\title{
Editorial
}

\section{Functional Oxide Thin Films and Nanostructures: Growth, Interface, and Applications}

\author{
Aiping Chen, ${ }^{1}$ Ying-Hao Chu, ${ }^{2}$ Run-Wei Li, ${ }^{3}$ Thomas Fix, ${ }^{4}$ and Jia-Mian $\mathrm{Hu}^{5}$ \\ ${ }^{1}$ Center for Integrated Nanotechnologies, Los Alamos National Laboratory, Los Alamos, NM 87545, USA \\ ${ }^{2}$ Department of Materials Science and Engineering, National Chiao Tung University, Hsinchu 30010, Taiwan \\ ${ }^{3}$ Key Lab of Magnetic Materials and Devices, Ningbo Institute of Material Technology and Engineering, \\ Chinese Academy of Sciences, Ningbo, Zhejiang 315201, China \\ ${ }^{4}$ Laboratoire ICube, CNRS-Université de Strasbourg, 67037 Strasbourg, France \\ ${ }^{5}$ Department of Materials Science and Engineering, Pennsylvania State University, University Park, PA 16802, USA \\ Correspondence should be addressed to Aiping Chen; apchen@lanl.gov
}

Received 6 December 2015; Accepted 5 January 2016

Copyright (C) 2016 Aiping Chen et al. This is an open access article distributed under the Creative Commons Attribution License, which permits unrestricted use, distribution, and reproduction in any medium, provided the original work is properly cited.

Functional oxides with novel physical/chemical properties have applications from optoelectronic devices, photocatalyst and information storage to biomedical applications, water splitting, and energy storage. The fascinating physical properties are strongly related to their electronic structures, surface morphology, interface, microstructure, defect, strain, and so forth [1-4]. Therefore, revealing the effect of these factors on functionalities is a key step to achieve practical applications of these oxides.

This special issue presents the synthesis, fabrication, and potential applications of metal oxide thin films and nanostructures. It was known that microstructure and strain play critical roles in physical properties in oxide thin films [5]. The research papers in this special issue further confirm that microstructures, surface morphology, interface, defect, and strain are connected with functionalities in different types of metal oxide thin films and nanostructures.

First set of papers are exploring the chemical synthesis of $\mathrm{ZnO}$ nanostructures to enhance the photocatalytic and photoelectrochemical reactions. For instance, N. A. Abd Samad et al. in their work titled "Easy Formation of NanodiskDendritic ZnO Film via Controlled Electrodeposition Process" highlighted the newly developed nanodisk-dendritic $\mathrm{ZnO}$ film, which can harvest more incident photons to generate more photoinduced charge carriers to trigger the photocatalytic and photoelectrochemical reactions. A.-J. Wang et al. in a paper titled "Facile Synthesis of Rambutan-Like
ZnO Hierarchical Hollow Microspheres with Highly Photocatalytic Activity" reported the improved photocatalytic activity for the degradation of rhodamine B under ultraviolet irradiation. After $90 \mathrm{~min}$ of UV irradiation, almost $100 \%$ of $\mathrm{RhB}$ molecules are decomposed for rambutan-like $\mathrm{ZnO}$ hierarchical hollow microspheres, unlike that of commercial $\mathrm{ZnO}$ with $23 \%$ of $\mathrm{RhB}$ molecules remaining. This work not only provides a simple method to prepare $\mathrm{ZnO}$ hollow structures but also sheds some light on the improvement of the photocatalytic performance by designing efficient catalysts.

Y. Liu et al. in a paper titled "Electrohydrodynamic Processing of p-Type Transparent Conducting Oxides" reported the synthesis of p-type transparent conducting oxide films such as $\mathrm{ZnO}$ and $\mathrm{CuAlO}_{2}$ thin films by electrospray electrospinning methods. The optical and electrical properties of these films have been carefully analyzed. The emerging applications of $\mathrm{CuAlO}_{2}$ as potential nanobuilding block have been discussed.

Defect significantly affects the properties in thin films and nanostructures. Oxygen vacancy is an important type defect in oxides [6]. R. Félix et al. in a paper titled "The Role of Edge Dislocations on the Red Luminescence of $\mathrm{ZnO}$ Films Deposited by RF-Sputtering" investigated the oxygen pressure dependence of structural and optical properties of $\mathrm{ZnO}$ films. The major defects have been identified as edge dislocations. The TEM characterization indicates that films 
deposited at low oxygen pressure present a higher density of threading dislocations. The red band shift has been found to correlate with the amount of edge dislocations.

C. L. Popa et al. in their work titled "Inhibitory Effect Evaluation of Glycerol-Iron Oxide Thin Films on MethicillinResistant Staphylococcus aureus" reported the inhibitory effect of glycerol-iron oxide thin films on methicillinresistant Staphylococcus aureus. The results suggest that glycerol-iron oxide thin films could be used in the future for various biomedical and pharmaceutical applications.

Interface is another important factor in metal/oxide multilayers [7]. H. Jiang et al. in a paper titled "Fabrication and Characterization of $\mathrm{Al} / \mathrm{NiO}$ Energetic Nanomultilayers" studied the redox reaction in $\mathrm{Al} / \mathrm{NiO}$ nanomultilayers. The interface effect was studied by deposited multilayers with different periodicity. It was found that the thermal diffusion time becomes greater as the amount of thermal boundary conductance across the interfaces increases with relatively smaller modulation period. Therefore, the interface of the $\mathrm{Al} / \mathrm{NiO}$ strongly influences the performance of energetic igniter.

Strain plays a critical role in controlling the growth and the properties of functional oxide thin films $[8,9]$. B. Ifland et al. in a paper titled "Strain Driven Phase Decomposition in Ion-Beam Sputtered $\operatorname{Pr}_{1-x} \mathrm{Ca}_{x} \mathrm{MnO}_{3}$ Films" used $\mathrm{Pr}_{1-x} \mathrm{Ca}_{x} \mathrm{MnO}_{3}$ films as a model system to study the influence of strain on the Mn solubility in perovskite oxide thin films. They observed that large tensile strain during deposition limits the Mn solubility of the perovskite phase. Mn excess gives rise to precipitates and the precipitation seems to represent a stress relaxation path.

The guest editors hope that this special issue can stimulate further research in the field of functional oxide thin films and nanostructures.

\section{Acknowledgments}

The editors gratefully thank the authors for their contributions to this special issue and the reviewers for their constructive comments.

Aiping Chen
Ying-Hao Chu
Run-Wei Li
Thomas Fix
Jia-Mian Hu

\section{References}

[1] V. T. Tra, J.-C. Yang, Y.-H. Hsieh, J.-Y. Lin, Y.-C. Chen, and Y.H. Chu, "Controllable electrical conduction at complex oxide interfaces," Physica Status Solidi-Rapid Research Letters, vol. 8, no. 6, pp. 478-500, 2014.

[2] T. Fix, E.-M. Choi, J. W. A. Robinson et al., "Electric-field control of ferromagnetism in a nanocomposite via a $\mathrm{ZnO}$ phase," Nano Letters, vol. 13, no. 12, pp. 5886-5890, 2013.

[3] J.-M. Hu, L.-Q. Chen, and C.-W. Nan, "Multiferroic heterostructures integrating ferroelectric and magnetic materials," Advanced Materials, vol. 28, no. 1, pp. 15-39, 2016.
[4] X. Zhu, F. Zhuge, M. Li et al., "Microstructure dependence of leakage and resistive switching behaviours in Ce-doped $\mathrm{BiFeO}_{3}$ thin films," Journal of Physics D: Applied Physics, vol. 44, no. 41, Article ID 415104, 2011.

[5] A. P. Chen, Z. X. Bi, Q. X. Jia, J. L. MacManus-Driscoll, and H. Y. Wang, "Microstructure, vertical strain control and tunable functionalities in self-assembled, vertically aligned nanocomposite thin films," Acta Materialia, vol. 61, no. 8, pp. 2783-2792, 2013.

[6] R. Zhao, W. W. Li, A. P. Chen et al., "Manipulating leakage behavior via distribution of interfaces in oxide thin films," Applied Physics Letters, vol. 105, no. 7, Article ID 072907, 2014.

[7] G. Radaelli, D. Petti, E. Plekhanov et al., "Electric control of magnetism at the $\mathrm{Fe} / \mathrm{BaTiO}_{3}$ interface," Nature Communications, vol. 5, article 3404, 2014.

[8] A. P. Chen, H. H. Zhou, Z. X. Bi et al., "A new class of room-temperature multiferroic thin films with bismuth-based supercell structure," Advanced Materials, vol. 25, no. 7, pp. 10281032, 2013.

[9] J. H. Haeni, P. Irvin, W. Chang et al., "Room-temperature ferroelectricity in strained $\mathrm{SrTiO}_{3}$," Nature, vol. 430, no. 7001, pp. 758-761, 2004. 

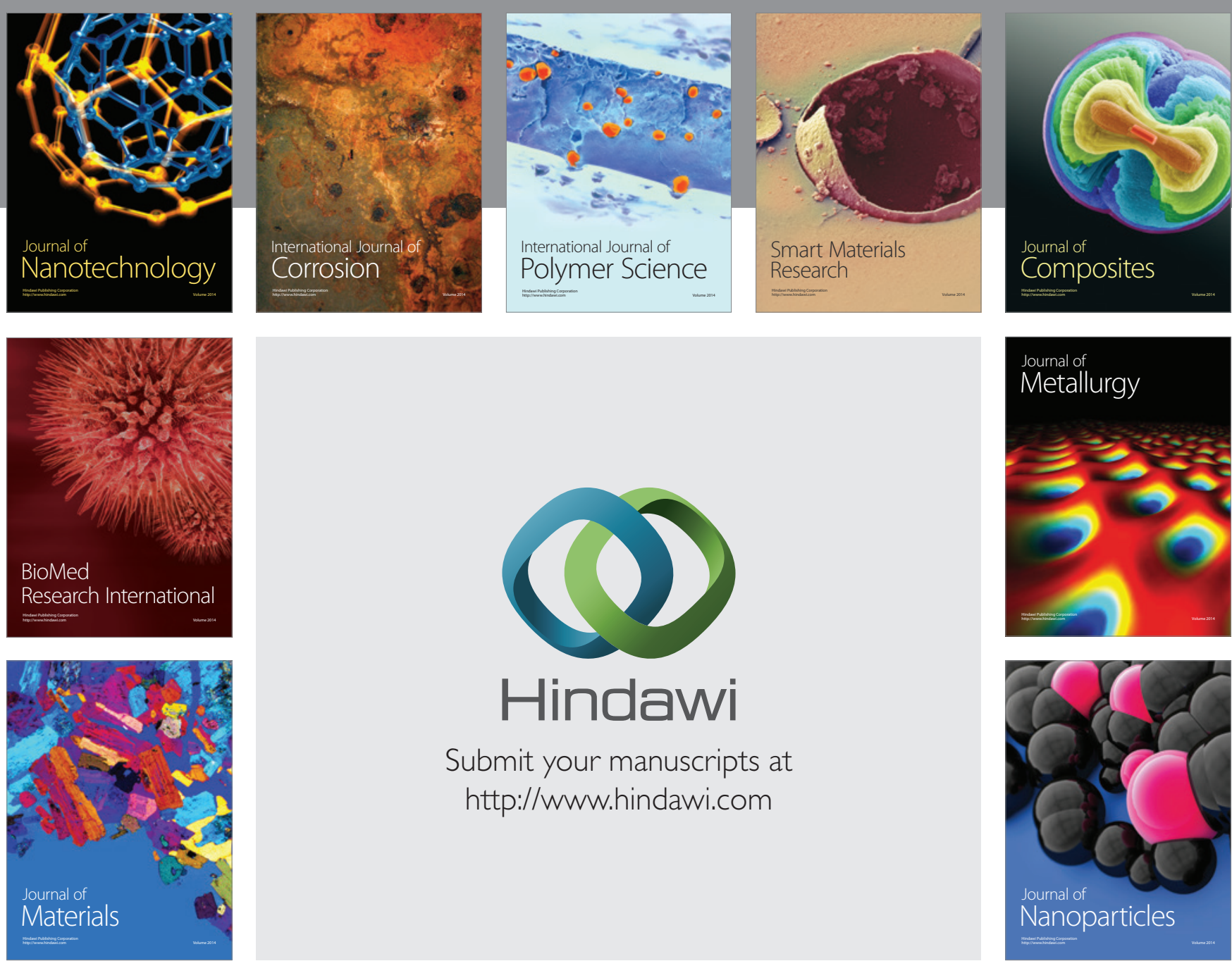

\section{Hindawi}

Submit your manuscripts at

http://www.hindawi.com

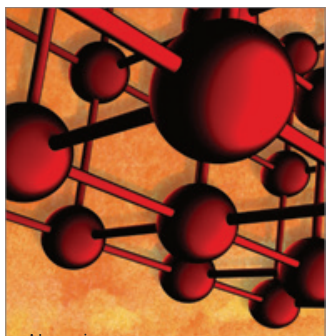

Materials Science and Engineering
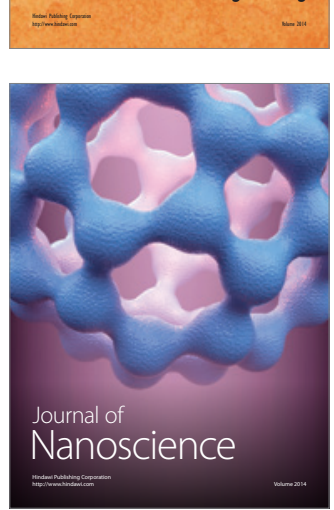
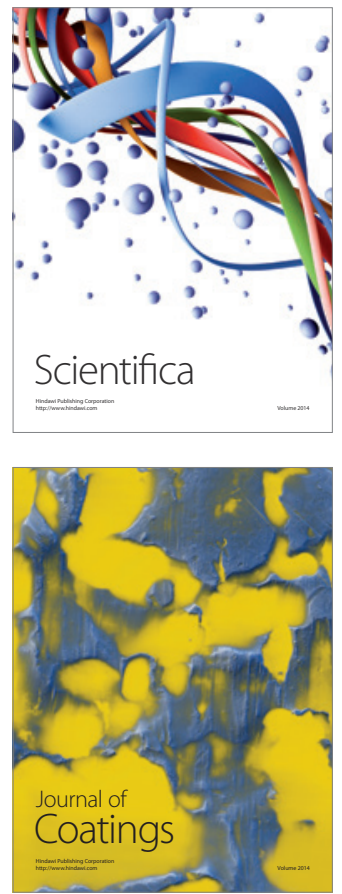
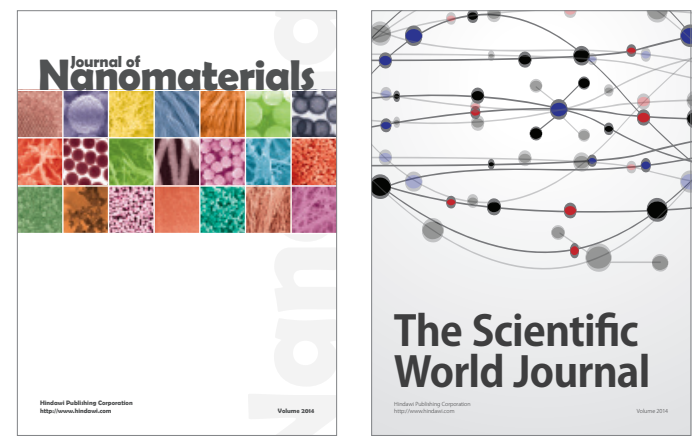

The Scientific World Journal
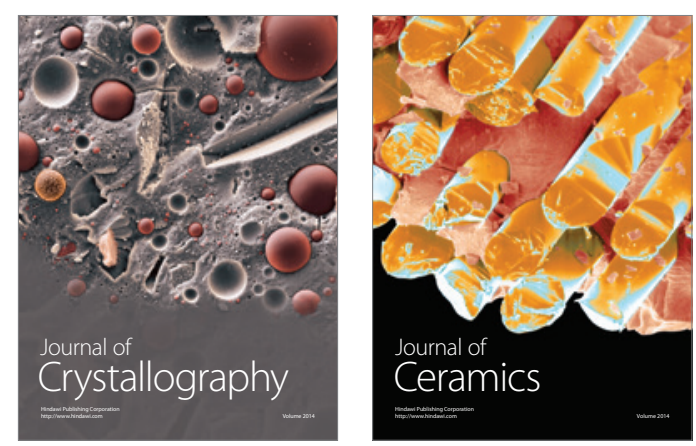
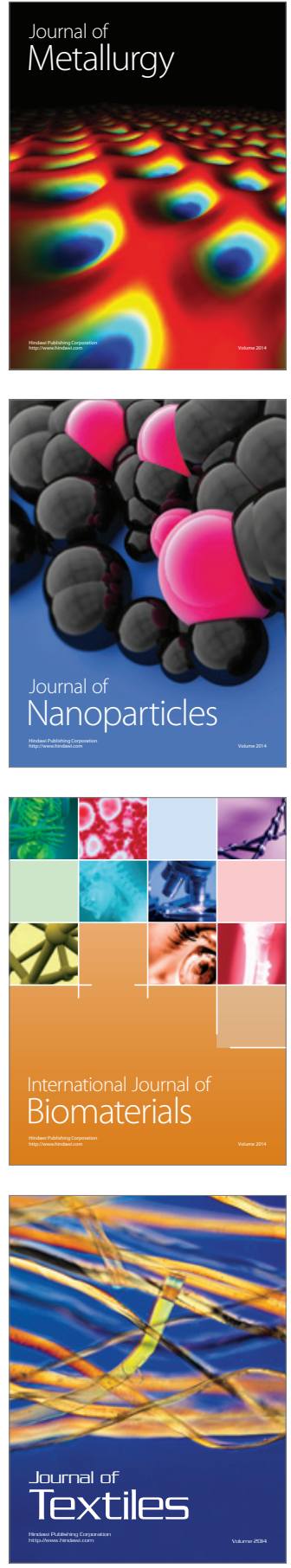